\title{
La FMH et la SSMG mènent une enquête sur l'équipement informatique des cabinets médicaux suisses (SISA 2007)
}

\author{
Marco Zoller ${ }^{a}$, Judith Wagner ${ }^{b}$, \\ Heinz Bhend', Franz Marty ${ }^{d}$ \\ a Unité de médecine de famille, \\ Université de Zurich \\ b responsable du Département \\ eHealth de la FMH \\ c responsable du groupe SSMG. \\ Informatics \\ d collaborateur du groupe SSMG \\ Informatics
}

\begin{abstract}
De quoi s'agit-il?
Fin 2006, la Confédération a mis en consultation une stratégie suisse en matière de cybersanté («e-health») et l'a approuvée en juin 2007 [1]. Puis elle a signé à ce sujet en septembre 2007 une convention-cadre avec les cantons. Cette stratégie prévoit un développement ambitieux qui concerne notamment les cabinets médicaux ambulatoires: d'ici à fin 2012, la majorité de ceux-ci devrait utiliser un extrait commun des dossiers électroniques personnels des patients et à fin 2015, ces dossiers devraient être à la disposition de tous les citoyens.

Aucun plan détaillé n'existe pour la mise en œuvre de cette vision. Ceci n'est pas étonnant car on ne dispose même pas, aujourd'hui, de données sur la situation actuelle et l'utilisation de l'infrastructure informatique des cabinets médicaux suisses.
\end{abstract}

\section{Le corps médical veut des faits comme base de discussion}

Dans ce contexte, la FMH et la SSMG ont décidé d'organiser une enquête représentative auprès de tous les médecins établis en cabinet. A cet effet, un questionnaire sera envoyé à environ un praticien sur onze.*

Ainsi donc, si ce questionnaire vous parvient au début novembre, nous vous prions instamment de le remplir puis de le renvoyer par fax. Pour que les résultats soient parlants, il est en effet essentiel que tous les questionnaires nous soient renvoyés. Leur évaluation sera effectuée par un institut professionnel neutre garantissant l'anonymat de vos données.

La FMH et la SSMG soutiennent en principe les mesures judicieuses relatives aux innovations technologiques au cabinet médical de leurs membres et s'intéressent depuis longtemps aux développements de la cybersanté [2-7]. Toutefois, avant de passer à la planification et à la mise en œuvre concrètes, il convient tout d'abord de clarifier en détail la situation actuelle ainsi que les conditions-cadre. Il apparaît que pour le passage exigé à la carte électronique de patient, de nombreux cabinets auraient besoin d'un soutien sous une forme ou une autre. Par conséquent, vos données revêtent une importance cruciale pour le corps médical, et ce, indépendamment de votre opinion positive ou totalement critique face aux changements annoncés.

Nous vous remercions d'ores et déjà de votre collaboration active à cette enquête, dont les résultats seront publiés dans le BMS.

\section{Références}

1 www.bag.admin.ch/themen/krankenversicherung/ 00305/03505/index.html?lang=fr.

2 Stellungnahme der FMH zur nationalen Strategie «eHealth»; 30 mars 2007. www.fmh.ch/ww/de/ $\mathrm{pub} / \mathrm{fmh} / \mathrm{standespolitik.htm} \mathrm{(en} \mathrm{allemand}$ uniquement).

3 Giger M, Wagner J. La carte de professionnel de santé des médecins suisses est en voie de réalisation. Bull Méd Suisses. 2007;88(33):1337-8.

4 Marty F, Bhend H, Kissling B, Späth HU. Vorschläge zur Expertentagung über eine nationale eHealthStrategie vom 11. Mai 2007 in Bern. PrimaryCare. 2007;7(18/19):315-6 (seul le résumé est traduit).

5 Bhend H. Nationale Strategie «eHealth» - Vision oder Illusion? PrimaryCare. 2007;7(18/19):314-5.

6 Bhend H. Praxisinformationssysteme der Zukunft. Bull Méd Suisses. 2007;88(35):1439-42 (en allemand uniquement).

7 Zürcher H, Metzger K. Positive Eindrücke vom dänischen Gesundheitssystem. Bull Méd Suisses. 2007;88(3):106-8 (en allemand uniquement). de 1200 membres FMH libres praticiens a été établi. 